\title{
A ILUSÓRIA DESCONCENTRAÇÃO INDUSTRIAL GAÚCHA APÓS A RECENTE CRISE ECONÔMICA
}

\author{
Antonio Paulo Cargnin ${ }^{1}$ \\ Bruno de Oliveira Lemos ${ }^{2}$ \\ Ana Maria de Aveline Bertê ${ }^{3}$ \\ Suzana Beatriz de Oliveira ${ }^{4}$
}

Resumo: $O$ presente artigo tem como finalidade analisar os efeitos da crise econômica brasileira, especialmente caracterizada no período 2012 a 2015, na distribuição territorial da indústria de transformação no Estado do Rio Grande do Sul. A hipótese central é de que a crise enfrentada pelo setor tem freado o processo de desconcentração que havia iniciado na década anterior, gerando uma tendência de inflexão no processo que havia conformado o chamado Eixo Expandido Porto Alegre-Caxias do Sul-Passo Fundo. As análises foram elaboradas tendo como base a utilização de dados de emprego fornecidos pela Relação Anual de Informações Sociais (RAIS), do Ministério do Trabalho e Emprego do Brasil (MTE), nos anos de 2012 a 2015.

Palavras-chave: Planejamento Territorial. Indústria de Transformação. Rio Grande do Sul. Desindustrialização. Crise econômica.

\section{THE ILLUSORY RIO GRANDE DO SUL INDUSTRIAL DECONCENTRATION AFTER THE RECENT ECONOMIC CRISIS}

Abstract: This article aims at analyzing the effects of the Brazilian economic crisis, mainly characterized in the period from 2012 to 2015, on territorial distribution of transformation industry in Rio Grande do Sul State. The central hypothesis is that the crisis faced by the sector has slowed down the process of deconcentration that had begun in the previous decade, thus generating an inflection trend in the process that had formed the so-called Porto Alegre-Caxias do Sul-Passo Fundo Expanded Axis. The employment data from 2012 to 2015, provided by the Annual Information of Social Information (RAIS) of the Ministry of Labor and Employment of Brazil (MTE), was the analyzes bases.

Keywords: Territorial Planning. Manufacturing Industry. Rio Grande do Sul State. Deindustrialization. Economic Crisis.

\section{LA ILUSÓRIA DESCONCENTRACIÓN INDUSTRIAL GAÚCHA DESPUÉS DE LA RECIENTE CRISIS ECONÓMICA}

\footnotetext{
${ }^{1}$ Secretaria de Planejamento, Governança e Gestão (SPGG), Departamento de Planejamento Governamental (DEPLAN); Programa de Pós-Graduação em Geografia da UFRGS (POSGEA/UFRGS), Porto Alegre, Brasil, antonio-cargnin@spgg.rs.gov.br; https://orcid.org/0000-0002-1212-0793

${ }^{2}$ Secretaria de Planejamento, Governança e Gestão (SPGG), Departamento de Planejamento Governamental (DEPLAN), Porto Alegre, Brasil, bruno-lemos@spgg.rs.gov.br, https://orcid.org/0000-0002-9865-4170

${ }^{3}$ Secretaria de Planejamento, Governança e Gestão (SPGG), Departamento de Planejamento Governamental (DEPLAN), Porto Alegre, Brasil, berte@spgg.rs.gov.br, https://orcid.org/0000-0002-2833-6572

${ }^{4}$ Secretaria de Planejamento, Governança e Gestão (SPGG), Departamento de Planejamento Governamental (DEPLAN), Porto Alegre, Brasil, suzana-oliveira@spgg.rs.gov.br, https://orcid.org/ 0000-0003-1349-7426
} 
Resumen: El presente artículo tiene como finalidad analizar los efectos de la crisis económica brasileña, especialmente caracterizada en el período 2012 a 2015, en la distribución territorial de la industria de transformación en el Estado de Rio Grande do Sul. La hipótesis central es que la crisis enfrentada por el sector ha frenado el proceso de desconcentración que había iniciado en la década anterior, generando una tendencia de inflexión en el proceso que había conformado el llamado Eje Expandido Porto Alegre-Caxias do Sul-Paso Fundo. Los análisis fueron elaborados teniendo como base la utilización de datos de empleo proporcionados por la Relación Anual de Información Social (RAIS), del Ministerio de Trabajo y Empleo de Brasil (MTE), en los años 2012 a 2015.

Palabras clave: Planificación Territorial. Industria de Transformación. Rio Grande do Sul. Desindustrialización. Crisis económica.

\section{Introdução}

A estruturação de um modelo de produção, a partir da expansão fabril no século XVIII, teve como resultado uma ocupação desigual do território. Essas disparidades se agravaram com a evolução do padrão global de produção e consumo, vigente nos dias atuais. Evidências desses distúrbios são observadas tanto do ponto de vista da desigualdade regional quanto do ponto de vista do uso do território e geram problemas de toda ordem. Deseconomias de aglomeração, problemas sociais, ambientais e migrações em massa são alguns dos resultados mais graves (HARVEY, 1993; ORY, 2008).

Nesse contexto, ganha relevância, especialmente no âmbito de atuação do Estado, a preocupação com o planejamento territorial. A ação do Estado, por meio do planejamento e do ordenamento territorial, contribui para a maior racionalidade da ocupação do território, minimizando os impactos indesejados e fortalecendo as racionalidades desejáveis ao desenvolvimento econômico e à melhoria da qualidade de vida das pessoas (GALIANA, VILUESA; 2010).

No Rio Grande do Sul, embora que de forma pouco efetiva, a preocupação com as disparidades regionais tem ocupado a agenda acadêmica e governamental. A preocupação com o tema se intensificou na década de 70 , quando estudos econômicos e demográficos já apontavam o declínio dos municípios do sul do Estado. Entretanto, é na segunda metade da década de 80 que o assunto começa a ocupar a agenda política e governamental, quando é estruturado o movimento em favor da chamada Metade Sul do Estado 5 .

O estudo denominado RS 2030 - Agenda de Desenvolvimento Territorial, finalizado em 2014, dá continuidade ao tema. Desenvolvido sob a coordenação da

5 Um balanço sobre essa temática pode ser encontrado em Cargnin (2011). 
Secretaria de Planejamento do Estado, os resultados chamaram a atenção para as principais dinâmicas territoriais vigentes no Rio Grande do Sul, especialmente as demográficas e as relativas à expansão das atividades econômicas (RIO GRANDE DO SUL, 2014).

O RS 2030 identificou a formação de um eixo de expansão industrial que se estende de municípios da Região Metropolitana de Porto Alegre (RMPA) para o noroeste do Estado, conformando o que pode ser chamado de Eixo Expandido Porto Alegre-Caxias do Sul-Passo Fundo. Além disso, observou o surgimento de um novo núcleo de expansão, em Rio Grande e Pelotas, associado ao polo naval que, na época, se estruturava. Entretanto, a forte retração recente da indústria de transformação, verificada nos últimos anos, tem lançado dúvidas sobre essa tendência. A esse respeito convém assinalar que a crise econômica enfrentada pelo País, associada a eventos mundiais e agravada pela crise política recente, afetou fortemente o segmento da indústria de transformação, sob o qual se assenta a indústria gaúcha.

Este artigo tem o objetivo de identificar elementos que indiquem mudanças no comportamento dessa dinâmica que havia conformado o chamado de Eixo Expandido Porto Alegre-Caxias do Sul-Passo Fundo. A tendência tinha sido evidenciada no artigo $A$ distribuição territorial da indústria de transformação no Estado do Rio Grande do Sul, utilizando dados da Relação Anual de Informações Sociais (RAIS), do Ministério do Trabalho e Emprego do Brasil (MTE), para os anos de 1995 a 2012 (LEMOS e CARGNIN, 2014).

A metodologia do presente artigo consistiu, primeiramente, no levantamento de dados de emprego da indústria de transformação do Rio Grande do Sul para o período de 2012 a 2015 no site da RAIS/MTE, considerando as divisões da Classificação Nacional de Atividades Econômicas (CNAE), do Instituto Brasileiro de Geografia e Estatística (IBGE). Essas divisões foram agrupadas conforme a tecnologia necessária para a produção dos bens, com base na classificação do Instituto de Estudos para o Desenvolvimento Industrial (IEDI, 2013). Posteriormente, foi realizada uma análise espacial dos dados de emprego da indústria de transformação do Rio Grande do Sul utilizando a cartografia temática.

A hipótese do artigo é de que a retração na indústria teria sido acompanhada de uma reversão da tendência de crescimento dos empregos dos segmentos de média e alta complexidade na indústria de transformação. Esse processo levaria à criação de um padrão de desconcentração ilusória, ou seja, que se restringe ao 
ganho de importância na indústria de segmentos tradicionais, especialmente agroalimentares, originalmente mais bem distribuídos.

\section{Principais Dinâmicas Territoriais no Estado}

A preocupação com as disparidades regionais no Estado, manifestada por meio de estudos acadêmicos e políticas governamentais nas últimas décadas, mostra o amplo desconforto com a concentração das atividades econômicas no território. Muitos são os estudos desenvolvidos, tanto no âmbito acadêmico quanto no das instituições governamentais, especialmente a partir da década de 90 (ALONSO, BANDEIRA, BENETTI; 1994; ALONSO, 2006; CARGNIN, 2011; FIORI, 2016).

Essa concentração fica evidente quando analisamos a distribuição da produção econômica, do emprego e da população. Se considerarmos a divisão regional dos Conselhos Regionais de Desenvolvimento (COREDEs) ${ }^{6}$, a partir de dados da Fundação de Economia e Estatística (FEE), para o ano de 2015, três regiões concentram cerca de $53 \%$ da produção econômica (PIB) e mais de $60 \%$ do valor de produção industrial, a saber: Metropolitano Delta do Jacuí, Vale do Rio dos Sinos e Serra.

A distribuição da população também segue a mesma lógica. Conforme dados do Censo Demográfico de 2010, elaborado pelo Instituto Brasileiro de Geografia e Estatística, as regiões Metropolitano Delta do Jacuí (22,6\%), Vale do Rio dos Sinos $(12,1 \%)$ e Serra $(8,1 \%)$, juntas, concentram $42,8 \%$ da população do Estado (CARGNIN, 2016).

Essa configuração se traduz em uma rede urbana hierarquizada a partir da Região Metropolitana de Porto Alegre (RMPA) e da Região Metropolitana da Serra Gaúcha (RMSG), conformando o chamado eixo Porto Alegre-Caxias do Sul, conforme pode ser observado na Figura 1. Entretanto, não se pode desconsiderar que o Rio Grande do Sul possui uma rede urbana bem articulada, contendo centros urbanos tradicionais de diferentes portes, como Santa Maria, Passo Fundo, e Pelotas e Rio Grande.

\footnotetext{
${ }^{6}$ Os COREDEs se constituem em um fórum de discussão e decisão sobre políticas e ações relativas ao desenvolvimento regional. Sua criação foi estimulada pelo Governo do Estado do Rio Grande do Sul a partir de 1991. A institucionalização se deu no ano de 1994. Atualmente, o Estado do Rio Grande do Sul é dividido em 28 COREDEs. Para assuntos mais amplos os COREDEs foram agrupados em 9 Regiões Funcionais de Planejamento, a partir do Estudo de Desenvolvimento Regional e Logística do RS - Rumos 2015 (RIO GRANDE DO SUL, 2006).
} 
Mesmo que em um contexto macrorregional a tendência de concentração continuasse, os dados recentes vinham apontando uma tênue desconcentração da atividade econômica e do emprego no eixo Porto Alegre-Caxias do Sul, expandindose na direção de Lajeado e Passo Fundo. Além disso, havia uma perspectiva de estruturação de um novo centro de desenvolvimento em torno do polo naval de Rio Grande.

Figura 1 - Taxa geométrica de crescimento anual da população 2000-2010, por COREDE e Rede de Cidades 2007

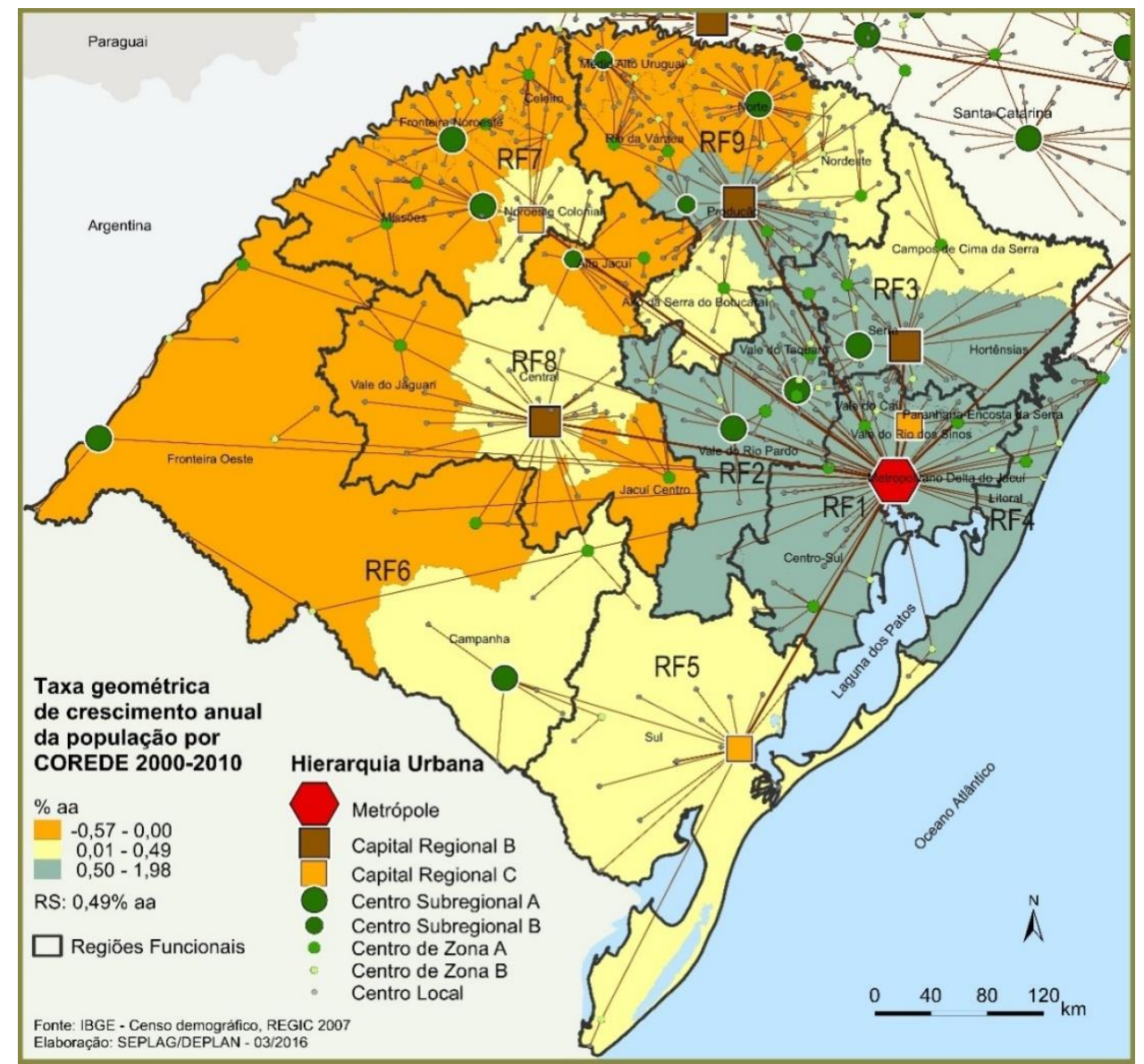

Fonte: IBGE - Censos Demográficos 2000 e 2010, REGIC 2007

Do ponto de vista demográfico, o grande destaque é a crescente perda de população do arco de fronteira, que vem apresentando seguidas taxas de crescimento negativas. Esse quadro conforma uma vasta área, que segue nossa fronteira com o Uruguai e Argentina e, também, os limites com Santa Catarina, como pode ser observado na Figura 1.

Considerando esses aspectos, a próxima seção procura avançar no sentido de compreender o deslocamento espacial da indústria a partir da crise exacerbada no período recente. Para tanto, é analisada a distribuição territorial dos empregos da 
indústria de transformação, sendo utilizados dados da Relação Anual de Informações Sociais (RAIS), do Ministério do Trabalho e Previdência Social, que são fornecidos pelos empregadores, restringindo-se aos empregos formais ${ }^{7}$.

\section{Distribuição territorial dos empregos da Indústria de Transformação no RS - 2012-2015}

De acordo com os dados disponíveis na RAIS/MTE, os empregos da indústria de transformação começaram a sofrer os impactos do processo de retração da economia nacional anteriormente em relação ao total de empregos formais no Rio Grande do Sul. O crescimento dos empregos da indústria de transformação no Estado ocorreu até 2013, regredindo nos anos seguintes, enquanto no valor total de empregos formais do Rio Grande do Sul esse crescimento se estendeu até 2014. A Tabela 1 demonstra o número de empregados na indústria de transformação e o total de empregos formais no Rio Grande do Sul e no Brasil e suas respectivas taxas de crescimento entre os anos de 2012 e 2015.

No período 2012-2015, o Rio Grande do Sul apresentou uma diminuição no número de empregados na indústria de transformação de $-7,76 \%$, pouco maior do que a média nacional, de $-7,34 \%$. No entanto, conforme se observa na Tabela 1, entre os anos de 2012-2013, a indústria de transformação estadual teve um crescimento no número de empregados de 1,97\%, pouco superior à brasileira, de $1,88 \%$.

Os primeiros impactos da crise econômica começam a aparecer entre os anos de 2013 e 2014, quando a taxa de crescimento no número de empregados na indústria de transformação no Rio Grande do Sul foi de $-2,13 \%$, enquanto a do Brasil foi de $-1,70 \%$. Entre 2014 e 2015, o aprofundamento da crise gera uma taxa de $-7,57 \%$ no Rio Grande do Sul e de $-7,47 \%$ no Brasil.

\footnotetext{
7 Uma importante ressalva é que, nos períodos de crise econômica, ocorre um aumento da informalidade, não detectada nos dados da RAIS.
} 
Tabela 1 - Número de empregados na Indústria de Transformação e no total da economia e taxas anuais de crescimento (2012-2015)

\begin{tabular}{|c|c|c|c|c|}
\hline & $\begin{array}{l}\text { Número de } \\
\text { empregados na } \\
\text { Indústria de } \\
\text { Transformação }\end{array}$ & $\begin{array}{c}\text { Taxa de crescimento } \\
\text { do número de } \\
\text { empregados na } \\
\text { Indústria de } \\
\text { Transformação (\%) } \\
\end{array}$ & $\begin{array}{l}\text { Número total de } \\
\text { empregados }\end{array}$ & $\begin{array}{l}\text { Taxa de crescimento } \\
\text { do número total de } \\
\text { empregados }(\%)\end{array}$ \\
\hline ano/período & 2012 & & 2012 & \\
\hline $\begin{array}{l}\text { Rio Grande do Sul } \\
\text { Brasil }\end{array}$ & $\begin{array}{r}708.846 \\
7.754 .545 \\
\end{array}$ & & $\begin{array}{r}2.993 .031 \\
47.458 .712 \\
\end{array}$ & \\
\hline ano/período & 2013 & $2012-2013$ & 2013 & 2012-2013 \\
\hline $\begin{array}{l}\text { Rio Grande do Sul } \\
\text { Brasil }\end{array}$ & $\begin{array}{r}722.805 \\
7.900 .136 \\
\end{array}$ & $\begin{array}{l}1,97 \\
1,88 \\
\end{array}$ & $\begin{array}{r}3.082 .991 \\
48.948 .433 \\
\end{array}$ & $\begin{array}{l}3,01 \\
3,14\end{array}$ \\
\hline ano/período & 2014 & $2013-2014$ & 2014 & 2013-2014 \\
\hline $\begin{array}{l}\text { Rio Grande do Sul } \\
\text { Brasil }\end{array}$ & $\begin{array}{r}707.391 \\
7.765 .846 \\
\end{array}$ & $\begin{array}{l}-2,13 \\
-1,70 \\
\end{array}$ & $\begin{array}{r}3.109 .179 \\
49.571 .510 \\
\end{array}$ & $\begin{array}{l}0,85 \\
1,27 \\
\end{array}$ \\
\hline ano/período & 2015 & $2014-2015$ & 2015 & 2014-2015 \\
\hline $\begin{array}{l}\text { Rio Grande do Sul } \\
\text { Brasil }\end{array}$ & $\begin{array}{r}653.835 \\
7.185 .512\end{array}$ & $\begin{array}{l}-7,57 \\
-7,47\end{array}$ & $\begin{array}{r}3.005 .549 \\
48.060 .807\end{array}$ & $\begin{array}{l}-3,33 \\
-3,05\end{array}$ \\
\hline
\end{tabular}

Fonte: RAIS/MTE

Segundo Bonelli e Pessôa (2010, p.20), a indústria é tipicamente um setor que produz bens elásticos em relação à renda, aumentando o peso na economia nacional nos momentos de crescimento econômico, ocorrendo o oposto em momentos de estagnação e/ou recessão. O mesmo motivo é apontado para as menores flutuações na produção de bens de consumo não duráveis, com menores variações em relação à renda quando comparados com bens intermediários e duráveis de consumo e de capital. Sendo assim, os bens de consumo não duráveis tendem a sofrer menos os impactos das crises econômicas.

O Eixo Porto Alegre-Caxias do Sul concentra o maior número de empregados na indústria de transformação do Rio Grande do Sul, também se destacando alguns centros regionais, como Erechim, Rio Grande, Lajeado, Santa Cruz do Sul e Passo Fundo. Essa concentração territorial da indústria de transformação no Rio Grande do Sul se constitui destacadamente no período 19501980 devido à perda de importância industrial da Metade Sul do Rio Grande do Sul e às mudanças na estrutura da Indústria do Estado nesse período, privilegiando as economias de aglomeração ${ }^{8}$ presentes no Eixo Porto Alegre-Caxias do Sul (LEMOS, OLIVEIRA, BANDEIRA, 2016; ALONSO, BANDEIRA, BENETTI, 1994). A

\footnotetext{
${ }^{8} \mathrm{O}$ conceito de economias de aglomeração foi formulado pelo economista inglês Alfred Marshall (1842-1924), e se refere a externalidades às empresas que favorecem sua aglomeração em determinadas regiões, como o acesso à mão de obra qualificada, à matéria-prima e as trocas de conhecimento entre essas empresas. O conceito de deseconomias de aglomeração está ligado a um processo de desconcentração dessas empresas a partir de fatores ligados à aglomeração excessiva, constituindo externalidades negativas, como o aumento do preço dos terrenos, as dificuldades de mobilidade e questões ambientais, como a poluição. Um exemplo desse processo é a Região Metropolitana de São Paulo que, a partir da década de 1970, começa a perder participação na produção industrial do País devido às deseconomias de aglomeração presentes na região.
} 
distribuição territorial dos empregos da indústria de transformação no Rio Grande do Sul, em 2015, quando ocorre o aprofundamento da crise da indústria de transformação brasileira, é demonstrada na Figura 2.

Entre os anos de 2013-2015, os municípios mais atingidos pela crise na indústria de transformação estavam localizados no Eixo Porto Alegre-Caxias do Sul. No período, os piores saldos em números absolutos no setor foram de Caxias do Sul, com -15.202 empregados; Porto Alegre, com -6.911 empregados; e Gravataí, com -6.548 empregados. Os COREDEs Metropolitano Delta do Jacuí, Vale do Rio dos Sinos e Serra que, em 2013, eram responsáveis por $54,58 \%$ dos empregos da indústria de transformação estadual, diminuem essa participação para 52,94\%.

Figura 2 - Empregados na Indústria de Transformação do RS - 2015

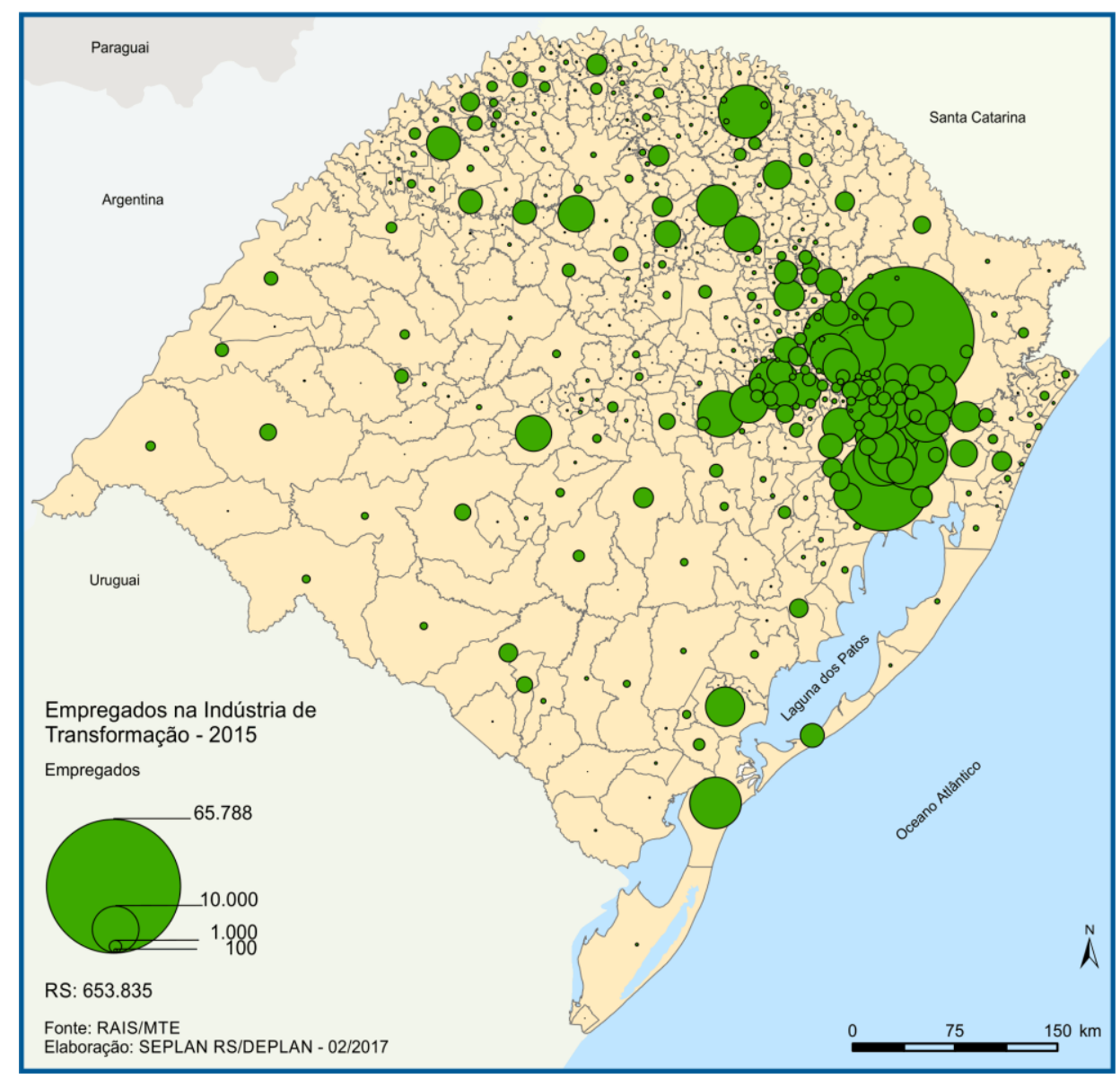

Fonte: RAIS/MTE

Na Figura 3, é possível observar que os municípios do Eixo Porto AlegreCaxias do Sul foram os que apresentaram piores saldos em empregos em números absolutos na indústria de transformação no período 2013-2015, quando se aprofunda a crise nesse setor. 
Figura 3 - Diferença no número de empregados na Indústria de Transformação 2013-2015

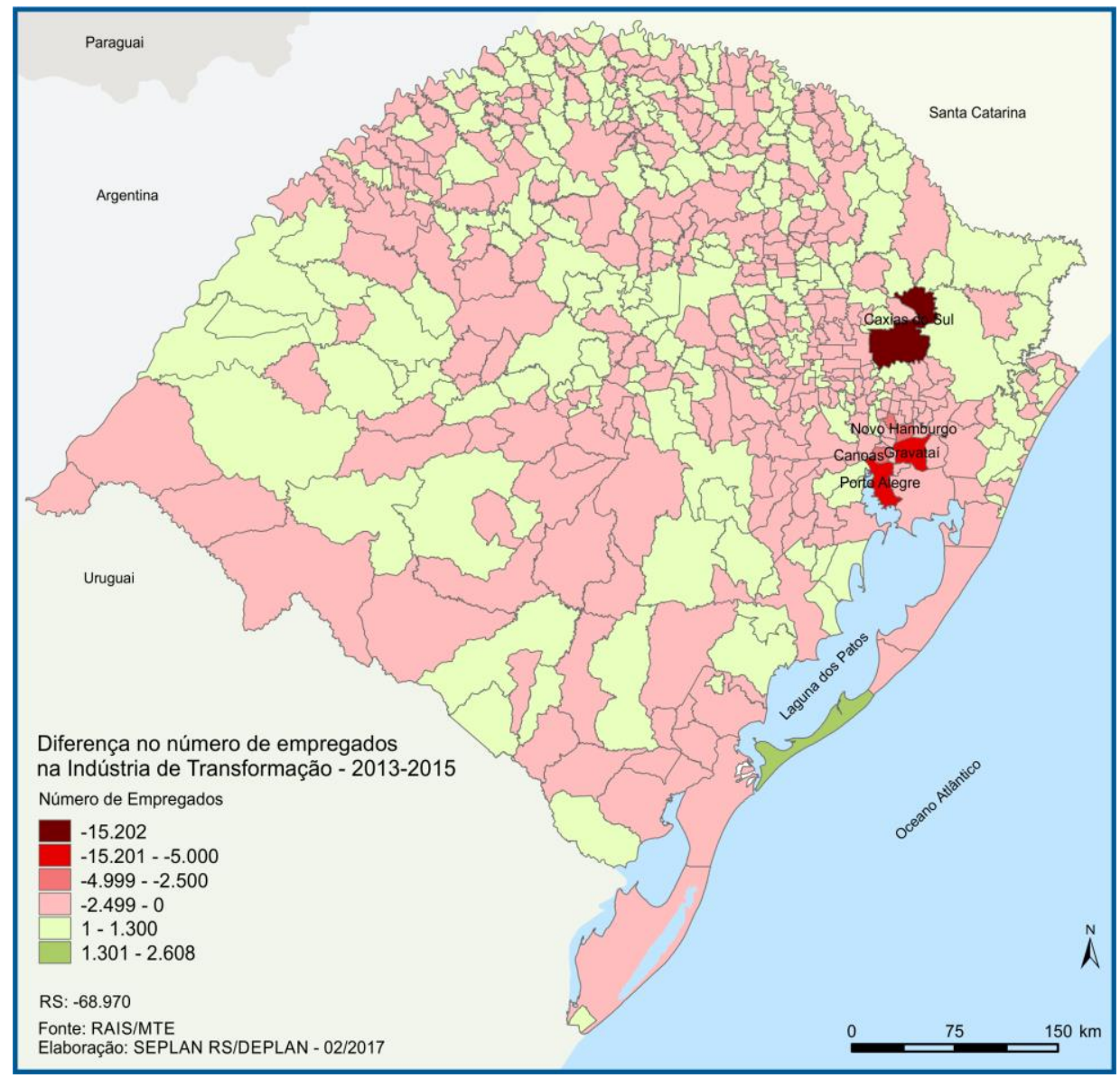

Fonte: RAIS/MTE

A diminuição nos números de emprego na indústria de transformação estadual, no período 2013-2015, é ainda mais intensa quando observamos os segmentos de alta e média-alta tecnologia, o que parece reforçar uma tendência de longo prazo da economia estadual de perda de participação industrial. Esses segmentos apresentam maiores capacidade de inovação e valor agregado à produção, estando mais ligados a uma mão de obra mais qualificada.

Conceição (2017), analisando os dados de Valor de Transformação Industrial (VTI), afirma que o atraso da indústria regional pode ser observado a partir da menor participação de setores de alta tecnologia e da perda de participação da indústria em relação ao País. No entanto, até 2013, observou-se um crescimento substancial de segmentos de média-alta tecnologia, como 0 de máquinas e equipamentos e o de fabricação de veículos automotores. Em 2014, a mudança da conjuntura nacional, com o aumento do desemprego, a queda na renda, a restrição 
de crédito às famílias e a diminuição do investimento, afetou o desempenho dos dois setores no Estado (CONCEIÇÃO, 2017).

Os segmentos de alta e média-alta tecnologia, segundo classificação presente na Carta IEDI n 569 (2013), podem ser considerados os seguintes:

Quadro 1 - Segmentos de alta e média-alta tecnologia da Indústria de Transformação

\begin{tabular}{|c|c|}
\hline \multirow{5}{*}{ Indústria de alta tecnologia } & Aeronáutica e espacial \\
\hline & Farmacêutica \\
\hline & Material de escritório e informática \\
\hline & $\begin{array}{l}\text { Equipamentos de rádio, } \\
\text { comunicação }\end{array}$ \\
\hline & $\begin{array}{l}\text { Instrumentos médicos de óptica e } \\
\text { precisão }\end{array}$ \\
\hline \multirow{5}{*}{$\begin{array}{l}\text { Indústria } \\
\text { tecnologia }\end{array}$} & Máquinas e equipamentos elétricos \\
\hline & $\begin{array}{l}\text { Veículos automotores, reboques e } \\
\text { semirreboques }\end{array}$ \\
\hline & Produtos químicos \\
\hline & $\begin{array}{l}\text { Equipamentos para ferrovia } \mathrm{e} \\
\text { material de transporte }\end{array}$ \\
\hline & Máquinas e equipamentos mecânicos \\
\hline
\end{tabular}

Fonte: Carta IEDI n 569 (2013)

Com base nessa classificação, as divisões da Classificação Nacional de Atividades Econômicas (CNAE 2.0) que abrangem os ramos de alta tecnologia da indústria de transformação podem ser consideradas: a) Fabricação de produtos farmoquímicos e farmacêuticos; e b) Fabricação de equipamentos de informática, produtos eletrônicos e ópticos. Já as de média-alta tecnologia podem abranger: a) Fabricação de produtos químicos; b) Fabricação de máquinas e equipamentos; c) Fabricação de máquinas, aparelhos e materiais elétricos; d) Fabricação de veículos automotores, reboques e carrocerias; e e) Fabricação de outros equipamentos de transporte, exceto veículos.

Os dados disponíveis na RAIS demonstram uma tendência de perda de participação dos segmentos de alta e média-alta tecnologia no número total de empregados na indústria de transformação estadual no período. Em 2013, a indústria de transformação estadual possuía 722.805 empregados, enquanto os segmentos de alta e média-alta tecnologia eram responsáveis por 180.198 
empregados, o que representava $24,93 \%$ do total do setor. Comparativamente, em 2015, a indústria de transformação do Rio Grande do Sul passou a deter 653.835 empregados, com as divisões de alta e média alta tecnologia possuindo 150.467, correspondendo a 23,01\%, ou seja, uma diminuição de participação desses segmentos de 1,92\% em relação a 2013.

Considerando as divisões da CNAE 2.0, podemos afirmar que os segmentos ligados ao metal-mecânico foram os mais atingidos pela crise no Rio Grande do Sul. A divisão Fabricação de Veículos Automotores, Reboques e Carrocerias possuía, em 2013, 55.368 empregados, passando a possuir 40.871 em 2015, uma perda 26,18\%; a de Fabricação de Máquinas e Equipamentos, em 2013, detinha 69.217 empregados, possuindo, em 2015, 57.522, com diminuição de -16,90\%; e a de Fabricação de Máquinas, Aparelhos e Materiais Elétricos apresentava, em 2013, 14.060 empregados, regredindo para 11.872 em 2015, o que representou diminuição de $-15,56 \%$. A Tabela 2 demonstra o número de empregados e as taxas anuais de crescimento no número de empregados desses segmentos entre 2012 e 2015 no Rio Grande do Sul e no Brasil.

Tabela 2 - Número de empregados e taxas de crescimento do número de empregados nas divisões da CNAE 2.0 Fabricação de Veículos Automotores, Reboques e Carrocerias, Fabricação de Máquinas e Equipamentos e Fabricação de Máquinas, Aparelhos e Materiais Elétricos (2012-2015)

\begin{tabular}{|c|c|c|c|c|c|c|c|}
\hline \multicolumn{8}{|c|}{ Empregados na Indústria de Transformação } \\
\hline \multirow[b]{2}{*}{ Ano/período } & \multicolumn{4}{|c|}{ número } & \multicolumn{3}{|c|}{ taxa de crescimento - \% } \\
\hline & 2012 & 2013 & 2014 & 2015 & 2012-2013 & 2013-2014 & 2014-2015 \\
\hline \multicolumn{8}{|c|}{ Fabricação de Veículos Automotores. Reboques e Carrocerias } \\
\hline Rio Grande do Sul & 52.103 & 55.368 & 49.391 & 40.871 & 6,27 & $-10,80$ & $-17,25$ \\
\hline Brasil & 514.483 & 532.364 & 489.279 & 426.107 & 3,48 & $-8,09$ & $-12,91$ \\
\hline \multicolumn{8}{|l|}{ Fabricação de Máquinas e Equipamentos } \\
\hline Rio Grande do Sul & 62.039 & 69.217 & 66.490 & 57.522 & 11,57 & $-3,94$ & $-13,49$ \\
\hline Brasil & 410.587 & 428.140 & 408.118 & 361.416 & 4,28 & $-4,68$ & $-11,44$ \\
\hline \multicolumn{8}{|c|}{ Fabricacão de Máquinas. Aparelhos e Materiais Elétricos } \\
\hline Rio Grande do Sul & 13.864 & 14.060 & 13.277 & 11.872 & 1,41 & $-5,57$ & $-10,58$ \\
\hline Brasil & 228.222 & 225.643 & 224.188 & 198.288 & $-1,13$ & $-0,64$ & $-11,55$ \\
\hline
\end{tabular}

Fonte: RAIS/MTE

Os segmentos ligados ao metal-mecânico se apresentam ainda mais concentrados no Eixo Porto Alegre-Caxias do Sul em relação aos outros, destacando-se nos COREDEs Metropolitano Delta do Jacuí, Vale do Rio dos Sinos e Serra. Contudo, estudos anteriores (RIO GRANDE DO SUL, 2014; LEMOS e CARGNIN, 2014) apontaram uma expansão dos empregos desses segmentos em direção ao noroeste do Estado do Rio Grande do Sul, configurando um Eixo 
Expandido Porto Alegre-Caxias do Sul ${ }^{9}$. A Figura 4 demonstra a concentração dos empregos de alta e média-alta tecnologia no Rio Grande do Sul em 2015.

Figura 4 - Empregados na Indústria de Transformação de alta e média alta tecnologia, por COREDE - 2015

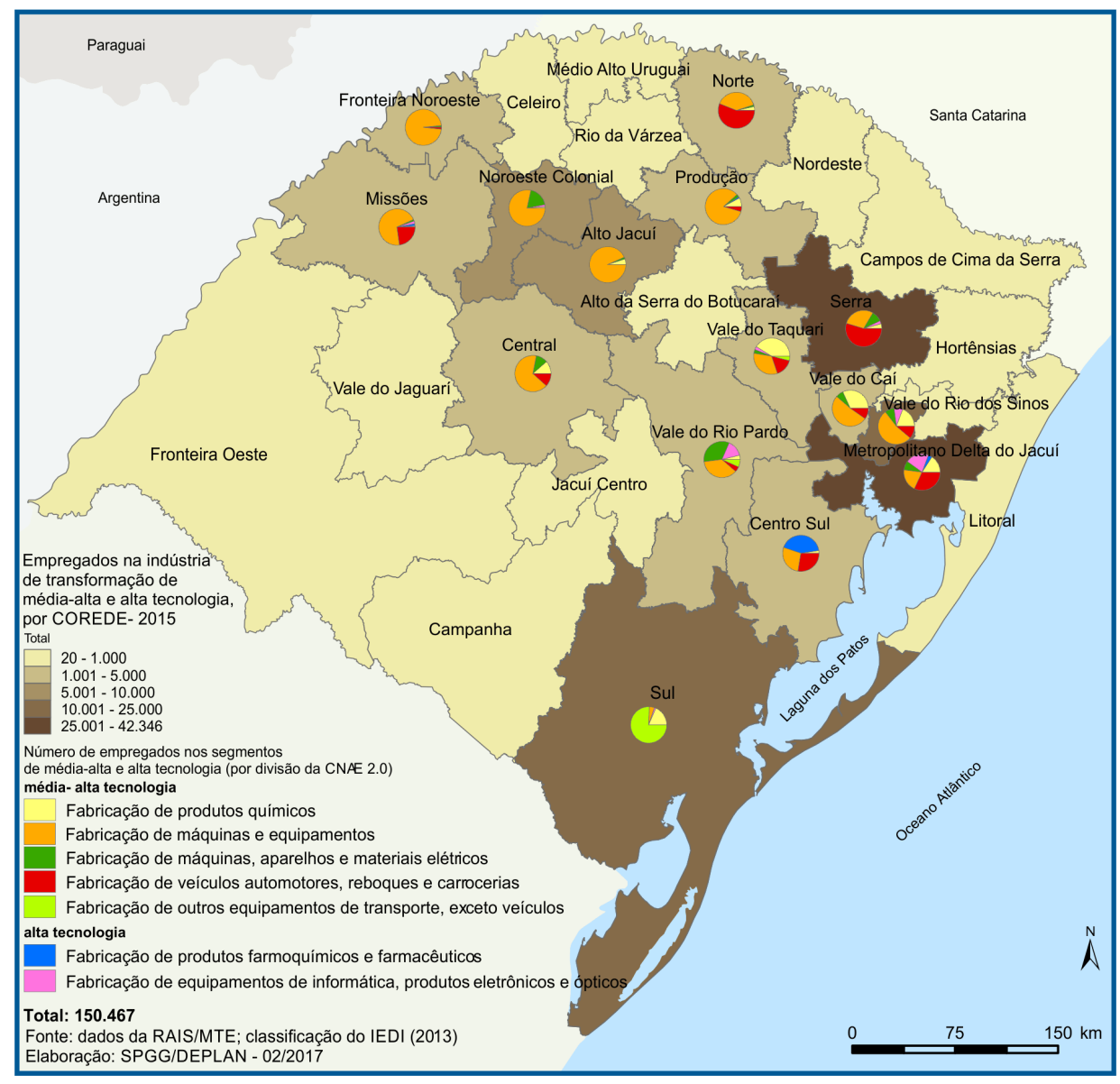

Fonte: RAIS/MTE

Por outro lado, alguns segmentos da indústria de transformação de menor tecnologia em sua produção e com importante representatividade no Estado detiveram perdas menores ou até cresceram nesse período. Como exemplo, a divisão da CNAE 2.0 de Fabricação de Produtos Alimentícios possuía, em 2013, 120.451 empregados, passando a deter, em 2015, 125.255, um crescimento de 3,99\%. Esses segmentos da indústria de transformação possuem maior distribuição territorial devido à menor dependência em relação às economias de aglomeração. $A$

\footnotetext{
${ }^{9}$ Outro fator responsável por uma relativa desconcentração da Indústria de Transformação, apontado em Cargnin et al (2014) e Cargnin Lemos (2014), é o crescimento da indústria naval, classificado na CNAE 2.0 na divisão de Fabricação de Outros Equipamentos de Transporte, exceto Veículos, podendo-se enquadrar entre as divisões de média-alta tecnologia. No entanto, observa-se que, em 2015, os impactos da crise econômica ainda não haviam aparecido nos dados de emprego disponíveis na RAIS. Por outro lado, notícias de dezembro de 2016 (JORNAL DO COMÉRCIO, 2016) confirmam demissões consideráveis no polo naval, o que pode representar a reversão de outro processo de desconcentração apontado no RS 2030: Agenda de Desenvolvimento Territorial.
} 
exceção no Estado constitui a indústria calçadista, concentrada nos COREDEs Vale do Rio dos Sinos e Paranhana Encosta da Serra.

Cano (2008), ao analisar o processo de desconcentração produtiva regional no Brasil no período 1970-2005, aponta para um processo de desconcentração virtuosa entre 1970 e 1980, como resultado dos projetos de larga envergadura implantados na periferia brasileira e das políticas de desenvolvimento regional implantadas a partir da metade de década de 1960. No entanto, o crescimento de São Paulo se mantinha nesse período. Em contraste, a partir da década de 1980, ocorre um fenômeno de desconcentração produtiva regional no Brasil muito mais pelos efeitos da crise econômica do período, que afetou os setores de maior tecnologia concentrados em São Paulo, do que por altas na produção periférica.

Observa-se, a partir de 2013, no Rio Grande do Sul, um processo semelhante em curto prazo, revertendo relativamente 0 processo de desconcentração virtuosa que ocorria anteriormente, com a expansão dos empregos na indústria de transformação estadual. Pode-se afirmar que, ao contrário dos processos de desconcentração da indústria identificados em Rio Grande do Sul (2014) e Lemos e Cargnin (2014), que se baseavam na expansão territorial de empregos de média-alta tecnologia ligados ao segmento metal-mecânico em direção ao norte-noroeste do Estado, a presente desconcentração parece estar ligada à crise econômica que, ao afetar esses segmentos, privilegia os de média-baixa e baixa tecnologia, já mais bem distribuídos no território do Rio Grande do Sul.

A Tabela 3 demonstra o número de empregados na indústria de transformação e as taxas anuais de crescimento do número de empregados nesse setor no período 2012-2015 por COREDE. Os COREDEs mais impactados pela crise econômica presente são os que possuem segmentos de média-alta e alta tecnologia, principalmente as divisões da CNAE 2.0 de Fabricação de Veículos Automotores, Reboques e Carrocerias, Fabricação de Máquinas e Equipamentos e Fabricação de Máquinas, Aparelhos e Materiais Elétricos. 
Tabela 3 - Número de empregados na Indústria de Transformação e taxas anuais de crescimento do número de empregados no setor por COREDE (2012-2015)

\begin{tabular}{|c|c|c|c|c|c|c|c|}
\hline \multirow{3}{*}{ COREDE } & \multicolumn{7}{|c|}{ Empregados na Indústria de Transformação } \\
\hline & \multicolumn{4}{|c|}{ número } & \multicolumn{3}{|c|}{ taxa de crescimento - $\%$} \\
\hline & 2012 & 2013 & 2014 & 2015 & $2012-2013$ & 2013-2014 & $2014-2015$ \\
\hline Alto da Serra do Botucaraí & 1.741 & 1.765 & 1.858 & 1.953 & 1,38 & 5,27 & 5,11 \\
\hline Alto Jacuí & 7.313 & 8.389 & 7.814 & 7.253 & 14,71 & $-6,85$ & $-7,18$ \\
\hline Campanha & 4.886 & 5.290 & 5.212 & 5.144 & 8,27 & $-1,47$ & $-1,30$ \\
\hline Campos de Cima da Serra & 2.710 & 2.696 & 2.640 & 2.498 & $-0,52$ & $-2,08$ & $-5,38$ \\
\hline Celeiro & 3.588 & 3.725 & 3.996 & 4.120 & 3,82 & 7,28 & 3,10 \\
\hline Central & 8.548 & 9.143 & 8.977 & 8.607 & 6,96 & $-1,82$ & $-4,12$ \\
\hline Centro Sul & 8.400 & 8.590 & 7.999 & 7.240 & 2,26 & $-6,88$ & $-9,49$ \\
\hline Fronteira Noroeste & 12.790 & 14.091 & 13.348 & 12.499 & 10,17 & $-5,27$ & $-6,36$ \\
\hline Fronteira Oeste & 6.827 & 6.698 & 6.921 & 7.182 & $-1,89$ & 3,33 & 3,77 \\
\hline Hortênsias & 13.915 & 13.745 & 13.148 & 12.925 & $-1,22$ & $-4,34$ & $-1,70$ \\
\hline Jacuí Centro & 4.172 & 4.380 & 4.318 & 3.603 & 4,99 & $-1,42$ & $-16,56$ \\
\hline Litoral & 5.950 & 6.276 & 6.488 & 6.253 & 5,48 & 3,38 & $-3,62$ \\
\hline Médio Alto Uruguai & 4.517 & 4.486 & 5.012 & 5.610 & $-0,69$ & 11,73 & 11,93 \\
\hline Metropolitano Delta do Jacuí & 98.657 & 100.126 & 96.320 & 84.299 & 1,49 & $-3,80$ & $-12,48$ \\
\hline Missões & 5.893 & 6.265 & 6.170 & 5.620 & 6,31 & $-1,52$ & $-8,91$ \\
\hline Nordeste & 7.885 & 8.445 & 8.290 & 8.573 & 7,10 & $-1,84$ & 3,41 \\
\hline Noroeste Colonial & 10.533 & 12.525 & 12.562 & 10.594 & 18,91 & 0,30 & $-15,67$ \\
\hline Norte & 17.541 & 18.930 & 18.050 & 16.671 & 7,92 & $-4,65$ & $-7,64$ \\
\hline Paranhana Encosta da Serra & 43.618 & 43.765 & 41.544 & 38.134 & 0,34 & $-5,07$ & $-8,21$ \\
\hline Produção & 21.143 & 21.468 & 21.445 & 19.416 & 1,54 & $-0,11$ & $-9,46$ \\
\hline Rio da Várzea & 4.545 & 4.904 & 4.935 & 4.814 & 7,90 & 0,63 & $-2,45$ \\
\hline Serra & 160.727 & 161.373 & 160.331 & 143.253 & 0,40 & $-0,65$ & $-10,65$ \\
\hline Sul & 26.385 & 26.289 & 26.938 & 25.612 & $-0,36$ & 2,47 & $-4,92$ \\
\hline Vale do Caí & 23.079 & 25.014 & 25.068 & 23.327 & 8,38 & 0,22 & $-6,95$ \\
\hline Vale do Jaguari & 1.963 & 2.060 & 2.159 & 2.101 & 4,94 & 4,81 & $-2,69$ \\
\hline Vale do Rio dos Sinos & 133.642 & 133.018 & 126.165 & 118.606 & $-0,47$ & $-5,15$ & $-5,99$ \\
\hline Vale do Rio Pardo & 24.830 & 25.100 & 25.362 & 24.206 & 1,09 & 1,04 & $-4,56$ \\
\hline Vale do Taquari & 43.048 & 44.249 & 44.321 & 43.722 & 2,79 & 0,16 & $-1,35$ \\
\hline Rio Grande do Sul & 708.846 & 722.805 & 707.391 & 653.835 & 1,97 & $-2,13$ & $-7,57$ \\
\hline
\end{tabular}

Fonte: RAIS/ MTE

Entre os anos de 2012 e 2013, os COREDEs com maior crescimento no número de empregos na indústria de transformação foram Noroeste Colonial, com 18,91\%; Alto Jacuí, com 14,71\%; e Fronteira Noroeste, com 10,17\%, enquanto a taxa estadual foi de 1,97\%, como demonstrado na Figura 5. Esse crescimento substancial refletia a expansão do número de empregos nas divisões da CNAE 2.0 de Fabricação de Máquinas e Equipamentos e de Fabricação de Máquinas, Aparelhos e Materiais Elétricos. Esse processo, que também se observava em um período mais longo, conduzia à expansão em direção ao noroeste do Eixo Porto Alegre-Caxias do Sul (RIO GRANDE DO SUL, 2014; LEMOS e CARGNIN, 2014). 
Figura 5 - Taxa de crescimento no número de empregados na Indústria de

Transformação - 2012-2013

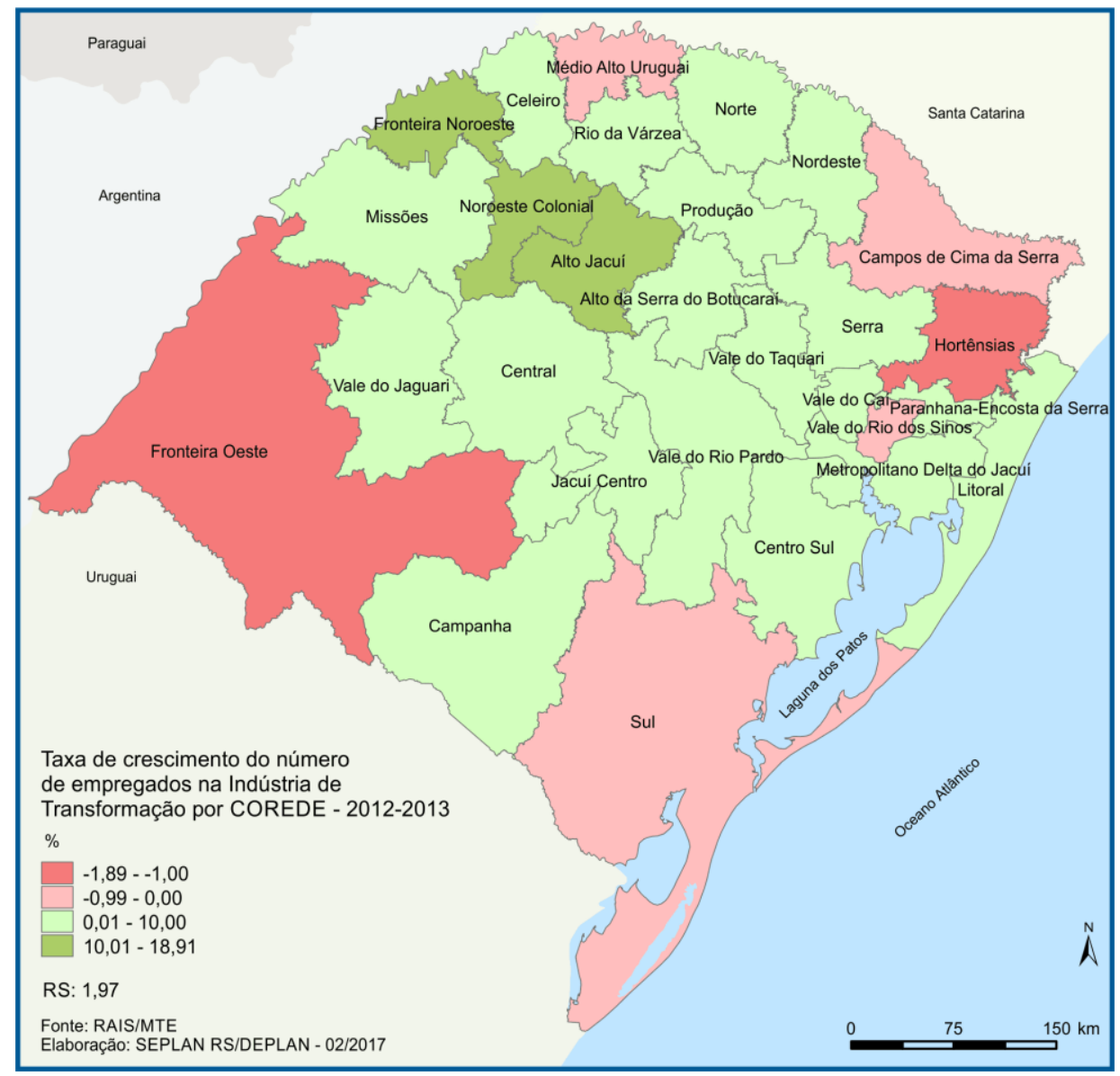

Fonte: RAIS/MTE

Contudo, após 2013, conforme demonstrado na Figura 6, a indústria de transformação estadual começa a sofrer os primeiros impactos da crise econômica, que atinge, especialmente, o segmento metal-mecânico, com considerável crescimento até esse período. Entre 2013 e 2015, observa-se uma diminuição substancial dos empregos da indústria de transformação em todos os COREDEs que apresentam esses segmentos. 
Figura 6 - Taxa de crescimento do número de empregados na Indústria de Transformação por COREDE - 2013-2015

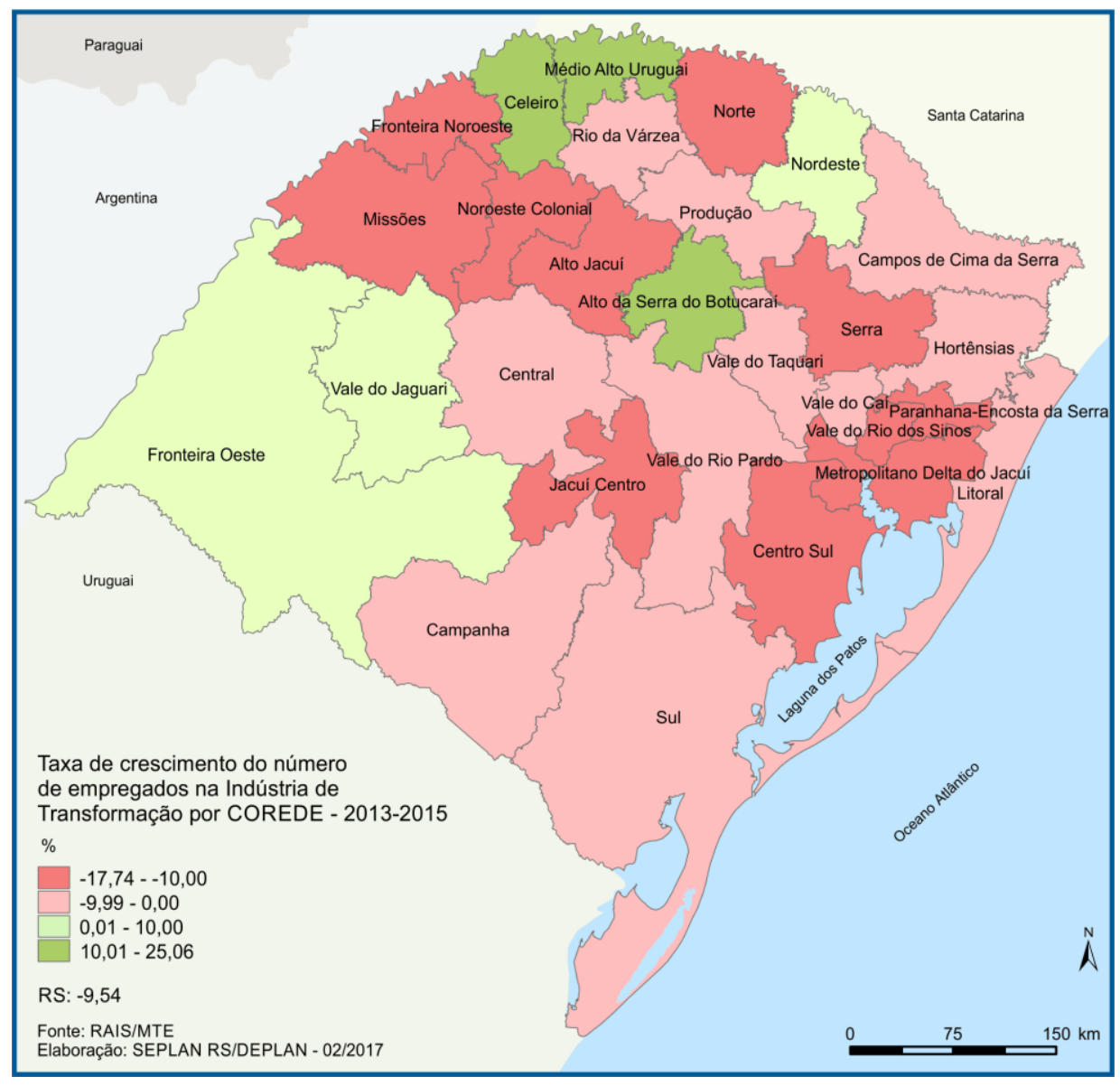

Fonte: RAIS/MTE

Esse período interrompe a expansão industrial dos segmentos de média-alta tecnologia no Estado, gerando, recentemente, uma relativa primarização ${ }^{10}$ da economia do Rio Grande do Sul. Esse processo se reflete nos ganhos de participação de regiões com matriz produtiva ligada à agropecuária e à

\footnotetext{
${ }^{10}$ Não é objetivo deste artigo discutir um suposto processo de desindustrialização pelo qual estaria passando a economia estadual, debatido em escala nacional por Bonelli e Pessôa (2010), Nassif (2008) e Oreiro e Feijó (2010). No entanto, Lemos e Cargnin (2014) atentam para um crescimento no número de empregos na indústria de transformação no Estado, entre 2000 e 2011, de 43,54\%, abaixo da geração de empregos formais total no período, de 54,22\%. Apesar do crescimento de ambos, isso representou uma diminuição de 27,15\%, em 2000, para 25,27\%, em 2011, da participação da indústria de transformação nos empregos formais do Estado. Oreiro e Feijó (2010, p.221) afirmam que a desindustrialização pode ocorrer mesmo quando há expansão industrial, pois se caracteriza pela perda de importância da indústria como fonte geradora de empregos e/ou de valor adicionado para uma determinada economia.
} 
agroindústria ${ }^{11}$, gerando uma desconcentração ilusória da economia do Rio Grande do Sul no período 2013-2015.

\section{Considerações Finais}

Embora apresente uma rede urbana relativamente equilibrada, o Rio Grande do Sul apresenta uma estrutura produtiva fortemente concentrada, o que se reflete na distribuição de sua população. Somente três COREDEs, que fazem parte do Eixo Porto Alegre-Caxias do Sul, concentram mais de $50 \%$ do PIB e $60 \%$ do VAB da Indústria do Estado. A dinâmica econômica se reflete na distribuição da população, com fluxos que se direcionam das demais regiões para esse Eixo em busca de oportunidades. Esse quadro impõe uma preocupação com o ordenamento territorial para evitar os problemas das economias de aglomeração e os decorrentes problemas ambientais e sociais. Por outro lado, desafia a implementação de políticas e instrumentos para a dinamização dos territórios que não se inserem nesse processo.

O comportamento da indústria gaúcha, em especial, da indústria de transformação, é uma peça importante para compreender essas dinâmicas. Na última década, a indústria gaúcha, alavancada pelo bom desempenho do segmento metal-mecânico, apresentou um deslocamento espacial, fomentando a desconcentração do emprego industrial. Esse fenômeno foi o principal elemento que atuou para a conformação do chamado Eixo Expandido Porto Alegre-Caxias do SulPasso Fundo.

A análise do comportamento do emprego industrial recente mostra que esse fenômeno perdura até o ano de 2013 quando, a partir de então, é drasticamente interrompido esse processo. A partir desse ano há uma diminuição significativa do emprego industrial nas regiões que apresentam maior participação desses segmentos. No caso do Rio Grande do Sul, isso repercute com muita ênfase nos segmentos industriais de média e alta tecnologia, que apresentavam uma tênue tendência de deslocamento espacial nos anos anteriores.

Por outro lado, os segmentos ligados à agropecuária e à agroindústria são menos afetados pela crise, contribuindo para uma possível primarização da economia gaúcha, com ganhos de participação de regiões com matriz produtiva ligada a esses segmentos. Como a produção agropecuária e a agroindústria a ela

\footnotetext{
${ }^{11}$ Entre os anos de 2013 e 2015, os COREDEs com maior crescimento percentual no número de empregados da indústria de transformação foram Médio Alto Uruguai, com 25,06\%; Alto da Serra do Botucaraí (10,65\%); Celeiro (10,60\%); e Fronteira Oeste (7,23\%).
} 
associada são tradicionalmente os mais bem distribuídos pelo território, observa-se uma desconcentração ilusória da indústria.

\section{REFERÊNCIAS}

ALONSO, José Antonio Fialho; BANDEIRA, Pedro Silveira; BENETTI, Maria Domingues. Crescimento econômico da região sul do RS: causas e perspectivas. Porto Alegre, FEE, 1994. 229p.

ALONSO, José Antonio Fialho. A persistência das desigualdades regionais no RS: velhos problemas, soluções convencionais e novas formulações. Indicadores Econômicos FEE, Porto Alegre, FEE, v.33, n.4, 2006. p. 101-114.

BONELLI, Regis; PESSÔA, Samuel de Abreu. Desindustrialização no Brasil: um resumo da evidência. Texto para Discussão IBRE/FGV, n.7, março de 2010.

CANO, Wilson. Desconcentração produtiva regional do Brasil - 1970-2005. São Paulo: Editora da UNESP. 2008.

CARGNIN, Antonio Paulo. Políticas de Desenvolvimento Regional no Rio Grande do Sul: Vestígios, Marcas e Repercussões Territoriais. Brasília, Ministério da Integração Nacional, 2011. 240p.

Desafios para o Planejamento e o Desenvolvimento Territorial no Estado do Rio Grande do Sul. Pelotas, Revista Geographia Meridionalis, v . 0 2, n. 02. 2016. p. 214233.

CONCEIÇÃO, César S. Evolução e dinâmica da indústria gaúcha e desempenho do sistema de inovação. In: Futuro RS: agenda de desenvolvimento: alternativas para a dinamização da indústria de transformação. Porto Alegre, Cadernos para o futuro 3, SPGG/DEPLAN. 2017. (No prelo).

FIORI, Tomás Pinheiro. Economia e Política do Desenvolvimento Regional no Rio Grande do Sul (1988-2010). Porto Alegre: Armazém Digital. 2016. 312 p.

GALIANA, L.; VINUESA, J. (Coords.) Teoría y práctica para una ordenación racional del territorio, Madrid, Síntesis, 2010.

HARVEY, D. Condição Pós-Moderna. São Paulo, Loyola, 1993. 349 p.

IEDI. Instituto de Estudos para o Desenvolvimento Industrial. Carta IEDI: n.569. 2013.

Disponível em: http://www.iedi.org.br/cartas/carta_iedi_n_569.html. Acesso em: 19.09.2018.

JORNAL DO COMÉRCIO. Demissões colocam em xeque o polo naval. Porto Alegre: 13.12.2016. Disponível em: http://jcrs.uol.com.br/_conteudo/2016/12/economia/536393demissoes-colocam-em-xeque-o-polo-naval.html. Acesso em: 05.05.2017.

LEMOS, Bruno de Oliveira; CARGNIN, Antonio Paulo. Características da distribuição territorial da indústria de transformação no estado do Rio Grande do Sul. Textos para Discussão FEE, n.126. p.1-23. 2014.

LEMOS, Bruno de Oliveira; OLIVEIRA, Suzana Beatriz de; BANDEIRA, Pedro Silveira. Agropecuária e indústria do Rio Grande do Sul no período 1920-1980: algumas 
características territoriais. Boletim Geográfico do Rio Grande do Sul, n.28. 2016.

Disponível em: http://revistas.fee.tche.br/index.php/boletim-geografico-

rs/issue/view/237/showToc. Acesso em: 05.05.2017.

OREIRO, José Luís; FEIJÓ, Carmem A. Desindustrialização: conceituação, causas, efeitos e o caso brasileiro. Revista de Economia Política, v.30, n.2. São Paulo: abril/junho 2010. Disponível em: http://www.scielo.br/pdf/rep/v30n2/03.pdf. Acesso em: 05.05.2017.

NASSIF, André. Há evidências de desindustrialização no Brasil? Revista de Economia Política, v.28, n.1. São Paulo: Jan./Mar. 2008.

ORY, Vicente Bielza de. Introducción a la ordenación del território: um enfoque geográfico. Zaragoza, Prensas Universitárias, Textos docientes, 2008. 280p.

RIO GRANDE DO SUL. Estudo de Desenvolvimento Regional e Logística para o Estado do Rio Grande do Sul - Rumos 2015 (Volumes 1, 2, 3, 4, 5 e Relatório Síntese). Porto Alegre, SCP, 2006.

RS 2030: Agenda de Desenvolvimento Territorial. Porto Alegre: Secretaria de Planejamento, Gestão e Participação Cidadã (SEPLAG) e Fundação de Economia e Estatística (FEE). 2014.

\section{NOTAS DE AUTOR}

\section{CONTRIBUIÇÃO DE AUTORIA}

Antonio Paulo Cargnin - Concepção. Coleta de dados. Análise de dados. Elaboração do manuscrito, revisão e aprovação da versão final do trabalho.

Bruno de Oliveira Lemos- Concepção e elaboração do manuscrito. Coleta de dados. Participação ativa da discussão dos resultados. Revisão e aprovação da versão final do trabalho.

Ana Maria de Aveline Bertê- Concepção e elaboração do manuscrito. Coleta de dados. Participação ativa da discussão dos resultados. Revisão e aprovação da versão final do trabalho.

Suzana Beatriz de Oliveira- Concepção. Coleta de dados. Análise de dados. Elaboração do manuscrito, revisão e aprovação da versão final do trabalho.

\section{FINANCIAMENTO}

Não se aplica.

\section{CONSENTIMENTO DE USO DE IMAGEM}

Não se aplica

\section{APROVAÇÃO DE COMITÊ DE ÉTICA EM PESQUISA}

Não se aplica.

\section{CONFLITO DE INTERESSES}

Não se aplica.

LICENÇA DE USO- uso exclusivo da revista

Este artigo está licenciado sob a Licença Creative Commons CC-BY. Com essa licença você pode compartilhar, adaptar, criar para qualquer fim, desde que atribua a autoria da obra.

HISTÓRICO- uso exclusivo da revista

Recebido em: 21-06-2017

Aprovado em: 24-09-2018 